\title{
Classified Ave Rage Precision For Capturing User Search Intention For Multiattributes
}

\author{
Anjuna D Raj , B.Ankayarkanni** \\ ${ }^{1,2}$ Dept of CSE Sathyabama University Chennai, India
}

\begin{abstract}
Due to the tremendous increase in use of internet surfing user may not get the most accurate search result which they preferred for their queries to clarify their known uncertain information. Such search engine may not often bring the user preferred information and does not satisfy the request completely. Hence it is necessary to infer and get user specific interest about a topic. Providing results just based on user's previous search history does not yield needful results since the self feedback and repeatable feedback were not included in the existing system. Those types of problems are solved in proposed system. So this proposal aims at the vigorous user feedback to provide accurate search specific results and to increase the performance of the search engine. This feedback is captured for all relevant URL that matches the search query using Classified Average Precision algorithm to yield most accurate web search results. Results from the clustered search are then categorized with captured feedback there by calculating the distance between the no of clicked links and user requested information in order to provide the exact information to the user. Likewise we can also search the image data based on the input which we giving as related image of our search result. Both images will be compared by calculating the histogramic values of images and the graph related to those values should be generated.
\end{abstract}

Keywords: Self-feedback,UserfeedbackHistogramic evalution,user search goals, adaptive similarity measure

\section{INTRODUCTION}

In search engines, normally queries are submitted to represent the needed information of the user. Sometimes such search engines does not retrieve the accurate information that the user who exactly meant since many ambiguous queries may hide a broad data and different users may want to get information on various aspects when they submit the similar query. . For example, when the query "the sun" is submitted to a search engine, some users want to locate the homepage of a United Kingdom newspaper, while some others want to learn the natural knowledge of the sun, as shown in. Therefore, it is necessary and potential to capture different user search goals in information retrieval. We define user search goals as the information on different aspects of a query that user groups want to obtain. Information need is a user's particular desire to obtain information to satisfy his/her need.

User search goals can be considered as the clusters of information needs for a query. The inference and analysis of user search goals can have a lot of advantages in improving search engine relevance and user experience. The proposed feedback session consists of both clicked and unclicked URLs and ends with the last URL that was clicked in a single session. It is motivated that before the last click, all the URLs have been scanned and evaluated by users.

Therefore, beside the clicked URLs, the unclicked ones before the last click should be a part of the user feedbacks. We can discover user search goals for some popular queries offline at first. Then, when users submit one of the queries, the search engine can return the results that are categorized into different groups according to user search goals online. Thus, users can find what they want conveniently

\section{SYSTEM STUDY}

\section{A. Feasibility Study}

The feasibility of the project is analyzed in this phase and business proposal is put forth with a very general plan for the project and some cost estimates. During system analysis the feasibility study of the proposed system is to be carried out. This is to ensure that the proposed system is not a burden to the company. For feasibility analysis, some understanding of the major requirements for the system is essential. Three key considerations involved in the feasibility analysis are

\section{A.1.Economical Feasibility}

This study is carried out to check the economic impact that the system will have on the organization. The amount of fund that the company can pour into the research and development of the system is limited. The expenditures must be justified. Thus the developed system as well within the budget and this was achieved because most of the technologies used are freely available. Only the customized products had to be purchased. 


\section{A.2.Technical Feasibility}

This study is carried out to check the technical feasibility, that is, the technical requirements of the system. Any system developed must not have a high demand on the available technical resources. This will lead to high demands on the available technical resources. This will lead to high demands being placed on the client. The developed system must have a modest requirement, as only minimal or null changes are required for implementing this system.

\section{A.3.Social Feasibility}

The aspect of study is to check the level of acceptance of the system by the user. This includes the process of training the user to use the system efficiently. The user must not feel threatened by the system, instead must accept it as a necessity. The level of acceptance by the users solely depends on the methods that are employed to educate the user about the system and to make him familiar with it. His level of confidence must be raised so that he is also able to make some constructive criticism, which is welcomed, as he is the final user of the system.

\section{HISTOGRAM REPRESENTATION}

Histogram equalization was a simple and effective image enhancing technique. But in some conditions, the importance of an image may be removed significantly only after the equalizing process, this is why it has never been utilized in a video system earlier. An image histogram equalization technique, equal area dualistic image histogram equalization, is put forward in our proposed work. First, the image is decomposed into two equal area sub-images based on its original probability density function. Then the two sub-images are equalized respectively. Finally, we obtain the results after the processed sub-images are composed into one image. The simulation results indicate that the algorithm can not only enhance the image information effectively but also preserve the original image clarity well enough to make it possible to be used.

Color histograms are widely used for content-based image retrieval. Their advantages are efficiency, and insensitivity to small changes in camera viewpoint. However, a histogram is a coarse characterization of an image, and so images with very different appearances can have similar histograms. We describe a technique for comparing images called histogram refinement, which imposes additional constraints on histogram based matching. Histogram refinement splits the pixels in a given bucket into several classes, based upon some local property. Within a given bucket, only pixels in the same class are compared. We describe a split histogram called a color coherence vector (CCV), which partitions each histogram bucket based on spatial coherence. CCVs can be computed at over 5 images per second on a standard workstation. A database with 15,000 images can be queried using CCVs in under 2 seconds. We demonstrate that histogram refinement can be used to distinguish images whose color histograms are indistinguishable

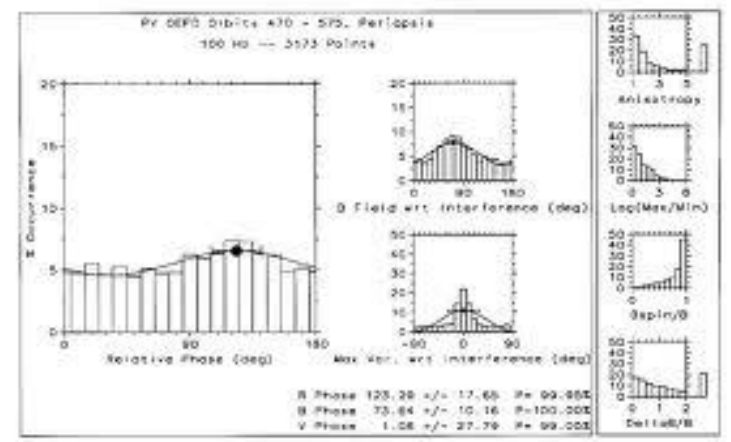

Fig 1. A sample histogram repesentation for images.

\section{FEEDBACK SESSION}

Feedback session is a type of session mainly used for information searching. Which is a sequence of related queries, inorder to satisfying an information needs. Some of the clicked results focused on find the user search goal for exact query. After that the single session it had simple one query, which differenciate information from the conservative query. the feedback session is based on solitary session though it may be comprehensive to the full session. It contains both unclicked and clicked URLs. Then it ends with the last URL which is clicked. It is forced that previous to the last click. All the URLs may be scanned and check by users.

Each feedback session denotes the user's most accurate and not needed information. Apart of these there are lots of diverse feedback sessions in user click-through logs. For inferring user search goals, it is additional effort to understand the feedback sessions than to examine the investigated clicked URLs in a straight 
line. To represent the feedback session efficiently some illustration methods are required, because every user based search goal feedback sessions are varied and their corresponding log files also changed.

Represent a feedback session to Pseudo-Documents with

Binary vector technique to characterize a feedback session

Search consequences are the URLs return by the search engine when the question "the sun" is submits, and "0" represent

"Unclicked" in the click sequence. The binary vector [0110001] can be second-hand to symbolize the feedback session, where " 1 " represent "clicked" and " 0 " represents "unclicked.

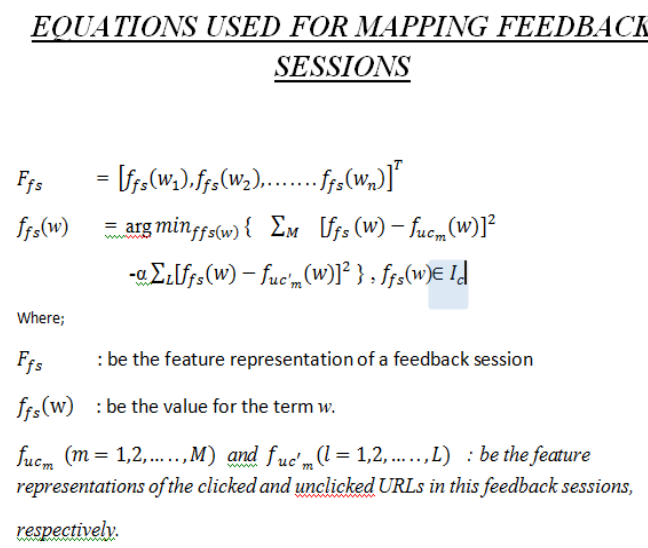

\section{BUILDING A PSEUDO-DOC}

The first step of this part is augmenting the initial URLs with additional text insided by retrieved topics and snippets of returned URLs displayed in the feedback session. Each URL in a feedback session is representing by a small text subsection that contain its heading and snippet. Also number of textual steps is implementing to person's text paragraphs, such as changing all the letters to lowercases, stemming and neglect stop words.

\section{RELATED WORKS}

Several approaches have been proposed in literature for textual as well as for image searching,

In Agglomerative clustering of a search engine query log

D. Beeferman and A. Berger, proposes the image retrieval in the text based and content based, including the newly growing ontology based image retrieval system as one focus. Semantic based image retrieval is an outstanding technique in retrieving the images from the image database. A multi dimensional concentration on the image extraction by providing weightage for various features like textual, colour, contrast, user feedback etc.., Request will be processed through mining engine, classification engine, segmentation engine and finally search engine. The use will get a perfect refined data, these are the merit of this paper

The demerits of this is since it's a survey paper, the rule base and fuzzy inference specified in the semantic based image retrieval is not clearly explained. Most of the things specified in this paper is concept based and there is no clear cut algorithm or specifications related to weight assignment operator, feature extraction and access formalities on image databases.

In Bringing Order to the Web: Automatically Categorizing Search Results, H. Chen and S. Dumais proposed that the active re-ranking of images in the web which is not available in the current system. Images will be parsed, manual ranking and user ranking is discussed in this project. It mainly focused on towards automation system and manual cum automated ranking option of the image is major focus of this project and it provides a fantastic perception about image ranking, this is the main merit. Didn't discuss properly on the non-class attributes properly and how we are segregating the class attributes based on the image attributes and image nature. This project didn't explain about the identification of symbolic images from the real images and didn't consider user's feedback for ranking the images. proposed the

In Context-Aware Query Suggestion by Mining Click-Through and Session Data, H. Cao and D. Jiang

Relevance feedback based interactive retrieval approach which selectively takes into account the two characteristics in CBIR.

And the merits of this paper is During the retrieval process the user high level query and perception subjectivity are captured by dynamically updated weights based on the users feedback these efforts have 
relatively ignored two distinct characteristics of CBIR systems The gap between high level concepts and low level features subjectivity of human perception of visual content. Truncation of multimedia content in this project is one of the major drawback. This paper emphasizes the usage of jpeg file and it remains a major drawback if in case of using different image types. This paper didn't explain the impact of using other image types.

In Query Recommendation using Query Logs in Search Engines, R. Baezza-Yates, C. Hurtado proposes Image feature representation, extraction, multi-dimensional indexing and system design are some of the terminologies focussed by the author for image textual retrieval. This paper explained the possible tools used in the content based textual retrieval area.

The merit of this paper is the authors have been focussed on the high level and lower level visual features of the images in the project. Focus on texture, shape, color layout, segmentation, indexing, dimension reduction is an add-on to the project. It's a general survey paper and not a implemented once. They didn't focus on any of the following things like security related part, logging related part, user feedback on the textual, image quality parts and finally performance part is the demerit.

In Relevant Term Suggestion in Interactive Web Search Based on Contextual Information in Query Session Logs, C.K. Huang, L. F. Chien and Y. I Oyang design a Conventional approaches to making term suggestions involve extracting co-occurring key terms from highly ranked retrieved documents. The relevant terms suggested for a user query are those that co-occur in similar query sessions from search engine logs, rather than in the retrieved documents. The merit of this paper is Not only do the search requests from the clients pass through the proxy server, but also all of the HTTP requests are the same. Partitioning the query log into query sessions accurately. Demerit is Log-based approach is not superior in every respect.

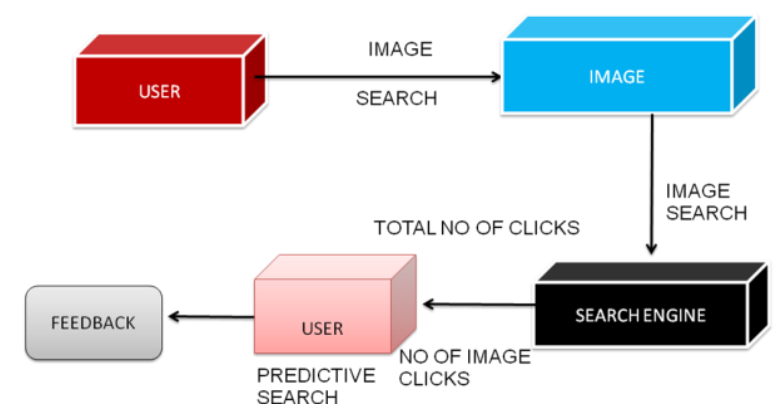

Fig 2. Shows the Data Flow Diagram

\section{B. METHODOLOGY}

To discovering the number of diverse user search goals for a query and depicting each goal with some keywords automatically. We first propose a novel approach to infer user search goals for a query by clustering our proposed feedback sessions. The feedback session is defined as the series of both clicked and unclicked URLs and ends with the last URL that was clicked in a session from user click-through logs. Then, we propose a novel optimization method to map feedback sessions to pseudo-documents which can efficiently reflect user information needs. At last, we cluster these pseudo documents to infer user search goals and depict them with some keywords.

\section{B.1. Textual Searching}

In this part, the search for the application has been carried by keyword based text search. The users are supposed to enter the keyword of search in the text format. Based on the keyword the list of websites with some explanations about the link will be displayed based on that user can choose the link.

This process will be finished with fraction of micro seconds.

\section{B.2. Site Re-Ranking}

One after the successful text search, the user clicks for the suggested URLs are tracked down. The feedback of the users for the site's are considered in this step. Based on the number of clicks and the feedback for the sites, the rank of the sites is modified. The ratings will be given based on the user review by using all these advanced options user might have easily choose the link.

\section{B.3. Image Uploading}

Here, image based search are formulated. The users are supposed to upload the image for their search. Based on the histogramic check of the image, relevant images are generated as the search result. From that user can choose the image same as before URL consideration in this image are listed in order. 


\section{B.4. Image Re-Ranking}

Once after the successful image search, the user clicks for the suggested URLs are tracked down. The feedback of the users for the sites is considered in this portion. Based on the number of clicks and the feedback for the sites, the rank of the sites are modified.

\section{B.5. Search Accuracy Check}

In this step, the accuracy in terms of search efficiency has been formulated. The relevant image fetch as the suggestion of the search from the input image are checked for its correctness.

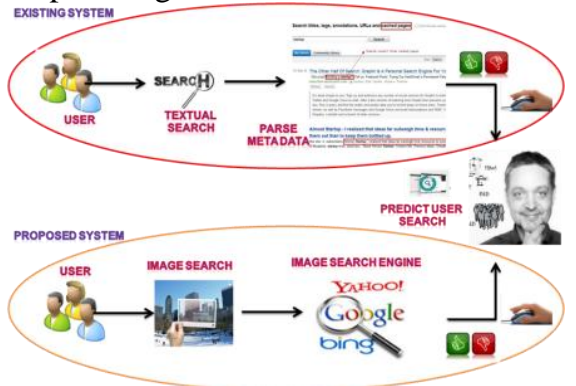

Fig 3. Shows the architecture diagram.

\section{CONCLUSION}

We suggested inferring user search goals for a query by clustering its feedback sessions represented by pseudo-documents. First, we introduce feedback sessions to be analyzed to infer user search goals rather than search results or clicked URLs. Both the clicked URLs and the unclicked ones before the last click are considered as user implicit feedbacks and taken into account to construct feedback sessions. Second, we map feedback sessions to pseudo-documents to approximate goal texts in user minds. our approach can discover user search goals for some popular queries offline at first. Then, when users submit one of the queries, the search engine can return the results that are categorized into different groups according to user search goals online.

\section{FUTURE ENHANCEMENT}

our approach can discover user search goals for some popular queries offline at first. Then, when users submit one of the queries, the search engine can return the results that are categorized into different groups according to user search goals online

\section{REFERENCE}

[1] R. Baeza-Yates and B. Ribeiro-Neto, Modern Information Retrieval.ACM Press, 1999. 512 IEEE TRANSACTIONS ON KNOWLEDGE AND DATA ENGINEERING, VOL. 25, NO. 3, MARCH 2013 Clustering of the clicked URLs and feedback sessions, where points represent clicked URLs, ellipses represent feedback sessions, and lines represent class boundaries. Supposing that the clicked URLs have two classes, the points in the left figure are hard to be segmented directly, while the ellipses in the right figure are easier to be segmented.

[2] R. Baeza-Yates, C. Hurtado, and M. Mendoza, "Query Recommendation Using Query Logs in Search Engines," Proc. Int'l Conf. Current Trends in Database Technology (EDBT '04), pp. 588-596, 2004.

[3] D. Beeferman and A. Berger, "Agglomerative Clustering of a Search Engine Query Log," Proc. Sixth ACM SIGKDD Int'l Conf. Knowledge Discovery and Data Mining (SIGKDD ’00), pp. 407-416, 2000.

[4] S. Beitzel, E. Jensen, A. Chowdhury, and O. Frieder, "Varying Approaches to Topical Web Query Classification," Proc. 30th Ann. Int'l ACM SIGIR Conf. Research and Development (SIGIR ’07), pp. 783-784, 2007.

[5] H. Cao, D. Jiang, J. Pei, Q. He, Z. Liao, E. Chen, and H. Li, "Context-Aware Query Suggestion by Mining Click-Through," Proc. 14th ACM SIGKDD Int'l Conf. Knowledge Discovery and Data Mining (SIGKDD '08), pp. 875-883, 2008.

[6] H. Chen and S. Dumais, "Bringing Order to the Web: Automatically Categorizing Search Results," Proc. SIGCHI Conf. Human Factors in Computing Systems (SIGCHI '00), pp. 145-152, 2000.

[7] C.-K Huang, L.-F Chien, and Y.-J Oyang, "Relevant Term Suggestion in Interactive Web Search Based on Contextual Information in Query Session Logs," J. Am. Soc. for Information Science and Technology, vol. 54, no. 7, pp. 638-649, 2003.

[8] T. Joachims, "Evaluating Retrieval Performance Using Click through Data," Text Mining, J. Franke, G. Nakhaeizadeh, and I. Renz, eds., pp. 79-96, Physica / Springer Verlag, 2003.

[9] T. Joachims, "Optimizing Search Engines Using Click through Data," Proc. Eighth ACM SIGKDD Int'l Conf. Knowledge Discovery and Data Mining (SIGKDD ’02), pp. 133-142, 2002.

[10] T. Joachims, L. Granka, B. Pang, H. Hembrooke, and G. Gay, "Accurately Interpreting Click through Data as Implicit Feedback," Proc. 28th Ann. Int'l ACM SIGIR Conf. Research and Development in Information Retrieval (SIGIR '05), pp. 154-161, 2005.

[11] R. Jones and K.L. Klinkner, "Beyond the Session Timeout: Automatic Hierarchical Segmentation of Search Topics in Query Logs," Proc. 17th ACM Conf. Information and Knowledge Management (CIKM '08), pp. 699-708, 2008.

[12] R. Jones, B. Rey, O. Madani, and W. Greiner, "Generating Query Substitutions," Proc. 15th Int'l Conf. World Wide Web (WWW '06), pp. 387-396, 2006

[13] U. Lee, Z. Liu, and J. Cho, "Automatic Identification of User Goals in Web Search,” Proc. 14th Int'l Conf. World Wide Web (WWW'05), pp. 391-400, 2005.

[14] X. Li, Y.-Y Wang, and A. Acero, "Learning Query Intent from Regularized Click Graphs," Proc. 31st Ann. Int'l ACM SIGIR Conf. Research and Development in Information Retrieval (SIGIR '08), pp. 339-346, 2008. 